\title{
On High Spatial Reuse Link Scheduling in STDMA Wireless Ad Hoc Networks
}

\author{
Ashutosh Deepak Gore, Srikanth Jagabathula and Abhay Karandikar
}

\begin{abstract}
Graph-based algorithms for point-to-point link scheduling in Spatial reuse Time Division Multiple Access (STDMA) wireless ad hoc networks often result in a significant number of transmissions having low Signal to Interference and Noise density Ratio (SINR) at intended receivers, leading to low throughput. To overcome this problem, we propose a new algorithm for STDMA link scheduling based on a graph model of the network as well as SINR computations. The performance of our algorithm is evaluated in terms of spatial reuse and computational complexity. Simulation results demonstrate that our algorithm achieves better performance than existing algorithms.
\end{abstract}

Index Terms-Wireless Ad hoc Networks, Spatial Time Division Multiple Access, Link Scheduling, Physical Interference Model, Spatial Reuse.

\section{INTRODUCTION}

A wireless ad hoc network consists of a finite number of radio units (nodes) that are geographically distributed in a terrain without any preplanned or fixed infrastructure. They communicate with each other via the untethered and broadcast wireless medium. In order to use the scarce and expensive wireless spectrum efficiently, we need to exploit channel spatial reuse, i.e., allow concurrent communication between source-destination pairs which are "reasonably" far from each other using either the same time slot or frequency band.

A commonly used scheme for channel reuse is Spatial Time Division Multiple Access (STDMA), in which time is divided into fixed-length slots that are organized cyclically. An STDMA schedule describes the transmission rights for each time slot in such a way that communicating pairs assigned to the same slot do not collide. STDMA scheduling algorithms can be categorized into link scheduling and broadcast/node scheduling algorithms [1]. In a wireless ad hoc network, a link is an ordered pair of nodes $(t, r)$, where $t$ is a transmitter and $r$ is a receiver. In link scheduling, the transmission right in every slot is assigned to certain links. On the other hand, in broadcast scheduling, the transmission right in every slot is assigned to certain nodes. Thus, there is no apriori binding of transmitter and receiver and the packet transmitted can be received by every neighbor. Link scheduling is suitable for unicast traffic, while broadcast scheduling is suitable for broadcast traffic. In this paper, we will concentrate on link scheduling for

A.D. Gore and A. Karandikar are with the Information Networks Laboratory, Department of Electrical Engineering, Indian Institute of Technology Bombay, Mumbai 400076, India. Email: \{adgore,karandi\}@ee.iitb.ac.in

S. Jagabathula is with the Laboratory for Information and Decision Systems, Massachusetts Institute of Technology, Cambridge, MA 02139, USA Email: jskanth@mit.edu
STDMA networks. Specifically, we consider centralized link scheduling, i.e., the link transmission schedule is computed by a central entity. Centralized scheduling is applicable for scenarios where the time scale of topology change is much larger than the duration of the schedule.

\section{A. Related Work}

The concept of STDMA for multihop wireless ad hoc networks was formalized in [2]. Centralized algorithms [3] [4] as well as distributed algorithms [5] [6] have been proposed for generating reuse schedules. The problem of determining an optimal minimum-length STDMA schedule for a general multihop ad hoc network is NP-complete for both link and broadcast scheduling [1]. In fact, this is closely related to the problem of determining the minimum number of colors to color all the edges (or vertices) of a graph under certain adjacency constraints. However, most wireless ad hoc networks can be modeled by planar or close-to-planar graphs and thus near-optimal edge coloring algorithms can be developed for these restricted classes of graphs.

A significant work in STDMA link scheduling is reported in [1], in which the authors show that tree networks can be scheduled optimally, oriented graphs can be scheduled nearoptimally and arbitrary networks can be scheduled such that the schedule is bounded by a length proportional to the graph thicknes 11 times the optimum number of colors.

A probabilistic analysis of the throughput performance of graph-based scheduling algorithms under the physical interference model is derived in [7]. The authors determine the optimal number of simultaneous transmissions by maximizing a lower bound on the physical throughput and subsequently propose a truncated graph-based scheduling algorithm that provides probabilistic guarantees for network throughput.

In [8], the authors present an analytical framework to investigate co-channel spatial reuse in dense wireless ad hoc networks based on path loss and log-normal shadowing models for a 1-D infinite regular chain topology and a 2$\mathrm{D}$ infinite hexagonally-tessellated topology. They derive the minimum ratio of inter-transmitter distance to transmitterreceiver distance, while still maintaining desirable Signal to Interference and Noise density Ratio (SINR) at the receivers. Their results demonstrate that increasing transmission power improves spatial reuse in ambient noise dominated environments. However, in co-channel interference limited scenarios, increasing transmission power has little effect on spatial reuse.

\footnotetext{
${ }^{1}$ The thickness of a graph is the minimum number of planar graphs into which the given graph can be partitioned.
} 
The performance of centralized graph-based and interference-based STDMA scheduling via simulations is evaluated and compared in [9]. To generate a graph-based conflict-free schedule, the authors use a two-level graph model with certain SINR threshold values chosen based on heuristics and examples. To generate an interference-based conflict-free schedule, the authors employ a method suggested in [10] which describes heuristics based on two path loss models, namely terrain-data based ground wave propagation model and Vogler's five knife-edge model.

In [11], the authors investigate throughput improvement in an 802.11-like wireless mesh network with Carrier Sense Multiple Access with Collision Avoidance (CSMA/CA) channel access scheme replaced by STDMA. For a successful packet transmission, they mandate that two-way communication be successful. Under this 'extended physical interference model', they present a greedy algorithm which computes the transmission schedule in a centralized manner. Assuming a uniform random node distribution and using results from occupancy theory, they derive an approximation factor for the length of this schedule relative to the shortest schedule.

Link scheduling for power-controlled STDMA networks under the physical interference model is analyzed in [12]. The authors define the scheduling complexity as the minimum number of time slots required for strong connectivity of the network. They develop an algorithm employing non-linear power assignment and show that its scheduling complexity is polylogarithmic in the number of nodes.

In a related work [13], the authors investigate the time complexity of scheduling a set of communication requests in an arbitrary network. They consider a 'generalized physical model' wherein the actual received power of a signal can deviate from the theoretically received power by a multiplicative factor. Their algorithm successfully schedules all links in time proportional to the squared logarithm of the number of nodes times the static interference measure [14].

In [15], the authors investigate the tradeoff between the average number of concurrent transmissions (spatial reuse) and sustained data rate per node for an 802.11 wireless network. Assuming that the channel data rate is given by the Shannon capacity, they show that spatial reuse depends only on the ratio of transmit power to carrier sense threshold. Keeping the carrier sense threshold fixed, they propose a distributed power and rate control algorithm based on interference measurement and evaluate its performance via simulations.

In [16], the authors investigate mitigation of inter-flow interference in an 802.11e wireless mesh network from a temporalspatial diversity perspective. Measurements of received signal strengths are used to construct a virtual coordinate system to identify concurrent transmissions with minimum inter-flow interference. Based on this new coordinate system, the gateway node determines the scheduling order for downlink frames of different connections. Through extensive simulation with reallife measurement traces, the authors demonstrate throughput improvement with their algorithms.

In [17], the authors consider wireless mesh networks with half duplex and full duplex orthogonal channels, wherein each node can transmit to at most one node and/or receive from at most $k$ nodes $(k \geqslant 1)$ during any time slot. They investigate the joint problem of routing flows and scheduling link transmissions to analyze the achievability of a given rate vector between multiple source-destination pairs. The scheduling problem is solved as an edge-coloring problem on a multigraph and the necessary conditions from scheduling problem lead to constraints on the routing problem, which is then formulated as a linear optimization problem. Correspondingly, the authors present a greedy coloring algorithm to obtain a 2 -approximate solution to the chromatic index problem and describe a polynomial time approximation algorithm to obtain an $\epsilon$-optimal solution of the routing problem using the primal dual approach. Finally, they evaluate the performance of their algorithms via simulations.

\section{B. Contributions of our Work}

In most STDMA algorithms, a link schedule is usually determined from a graph model of the network [1] [7] [9]. However, graph-based scheduling algorithms assume a limited knowledge of the interference and result in low network throughput. On the other hand, SINR-based scheduling algorithms [11] [12] [13] [15] require a complete knowledge of the interference and lead to higher throughput. Existing literature on SINR-based STDMA link scheduling consider system models which are different from our system model. For example, [11] [15] consider a variant of 802.11 wireless networks, [16] [17] consider wireless mesh networks and [12] [13] assume non-uniform transmit power at all nodes.

In this paper, we consider an STDMA wireless ad hoc network with uniform transmit power at all nodes and propose a link scheduling algorithm based on the graph model as well as SINR computations. We introduce spatial reuse as an important performance metric and argue that a high value of spatial reuse directly translates to high long-term network throughput. We show that the proposed algorithm has low computational complexity and high spatial reuse compared to existing algorithms.

The rest of the paper is organized as follows. In Section [II. we describe our system model along with the physical and protocol interference models, discuss the limitations of graphbased scheduling algorithms, formulate the problem and summarize the differences between our work and existing work in SINR-based scheduling algorithms. Section [III describes the proposed link scheduling algorithm. The performance of our algorithm is evaluated in Section IV and its computational complexity is derived in Section $\nabla$ We conclude and suggest directions for future work in Section VI

\section{SySTEM MOdeL}

Consider an STDMA wireless ad hoc network with $N$ static nodes (wireless routers) in a two-dimensional plane. During a time slot, a node can either transmit, receive or remain idle. We assume homogeneous and backlogged nodes. Let: 


$$
\begin{aligned}
\left(x_{j}, y_{j}\right) & =\text { Cartesian coordinates of } j^{t h} \text { node }=: \mathbf{r}_{j} \\
P & =\text { transmission power of every node } \\
N_{0} & =\text { thermal noise density } \\
D(j, k) & =\text { Euclidean distance between nodes } j \text { and } k
\end{aligned}
$$

We do not consider fading and shadowing effects. The received signal power at a distance $D$ from the transmitter is given by $\frac{P}{D^{\alpha}}$, where $\alpha$ is the path loss factor.

A link schedule effectively assigns sets of links to time slots. Specifically, a link schedule for the STDMA network is denoted by $\Psi\left(C, \mathcal{S}_{1}, \cdots, \mathcal{S}_{C}\right)$, where

$$
\begin{aligned}
C= & \text { number of slots in the link schedule } \\
\mathcal{S}_{i}= & \text { set of transmitter-receiver pairs which can } \\
& \text { communicate concurrently in the } i^{t h} \text { slot } \\
:= & \left\{t_{i, 1} \rightarrow r_{i, 1}, \cdots, t_{i, M_{i}} \rightarrow r_{i, M_{i}}\right\}
\end{aligned}
$$

where $t_{i, j} \rightarrow r_{i, j}$ denotes a packet transmission from node $t_{i, j}$ to node $r_{i, j}$ in the $i^{t h}$ slot. Note that $t_{i, j}, r_{i, j} \in\{1, \ldots, N\}$ and $M_{i}=\left|\mathcal{S}_{i}\right|$. The SINR at receiver $r_{i, j}$ is given by

$$
\operatorname{SINR}_{r_{i, j}}=\frac{\frac{P}{D^{\alpha}\left(t_{i, j}, r_{i, j}\right)}}{N_{0}+\sum_{\substack{k=1 \\ M_{i} \\ k \neq j}}^{\frac{P}{D^{\alpha}\left(t_{i, k}, r_{i, j}\right)}}}
$$

We define the signal to noise ratio (SNR) at receiver $r_{i, j}$ by

$$
\mathrm{SNR}_{r_{i, j}}=\frac{P}{N_{0} D^{\alpha}\left(t_{i, j}, r_{i, j}\right)}
$$

\section{A. Physical and Protocol Interference Models}

According to the physical interference model [18], $t_{i, j} \rightarrow$ $r_{i, j}$ is successful if and only if (iff) the SINR at receiver $r_{i, j}$ is greater than or equal to a certain threshold $\gamma_{c}$, termed as the communication threshold.

$$
\frac{\frac{P}{D^{\alpha}\left(t_{i, j}, r_{i, j}\right)}}{N_{0}+\sum_{\substack{k=1 \\ k=1 \\ k \neq j}}^{D^{\alpha}\left(t_{i, k}, r_{i, j}\right)}} \geqslant \gamma_{c}
$$

According to the protocol interference model [18], $t_{i, j} \rightarrow r_{i, j}$ is successful if:

1) the SNR at receiver $r_{i, j}$ is no less than the communication threshold $\gamma_{c}$. From (2), this translates to

$$
D\left(t_{i, j}, r_{i, j}\right) \leqslant\left(\frac{P}{N_{0} \gamma_{c}}\right)^{\frac{1}{\alpha}}=: R_{c}
$$

where $R_{c}$ is termed as communication range.

2) the signal from any unintended transmitter $t_{i, k}$ is received at $r_{i, j}$ with an SNR less than a certain threshold $\gamma_{i}$, termed as the interference threshold. This translates to

$$
\begin{aligned}
D\left(t_{i, k}, r_{i, j}\right) \geqslant\left(\frac{P}{N_{0} \gamma_{i}}\right)^{\frac{1}{\alpha}} & =: R_{i} \\
\forall k & =1, \ldots, M_{i}, k \neq j
\end{aligned}
$$

\footnotetext{
${ }^{2} \mathrm{~A}$ node is generically denoted by $j, j=1, \ldots, N$. However, we have used the notation $t_{i, j}$ to denote a node transmitting in the $i^{\text {th }}$ slot. Similarly, a node receiving in the $i^{\text {th }}$ slot is denoted by $r_{i, j}$.
}

where $R_{i}$ is termed as interference range. Note that $0<$ $\gamma_{i}<\gamma_{c}$, thus $R_{i}>R_{c}$.

The physical model of our system is denoted by $\Phi\left(N,\left(\mathbf{r}_{1}, \ldots, \mathbf{r}_{N}\right), P, \gamma_{c}, \gamma_{i}, \alpha, N_{0}\right)$.

A schedule $\Psi(\cdot)$ is feasible if it satisfies the following:

1) Operational constraint: A node must not perform multiple operations in a single time slot.

$$
\begin{aligned}
\left\{t_{i, j}, r_{i, j}\right\} \cap\left\{t_{i, k}, r_{i, k}\right\}=\phi & \forall i=1, \ldots, C \\
& \forall 1 \leqslant j<k \leqslant M_{i}
\end{aligned}
$$

2) Communication range constraint: Every receiver is within the communication range of its intended transmitter.

$$
D\left(t_{i, j}, r_{i, j}\right) \leqslant R_{c} \forall i=1, \ldots, C \forall j=1, \ldots, M_{i}
$$

A schedule $\Psi(\cdot)$ is exhaustive if it satisfies the following:

$$
\begin{array}{r}
D(j, k) \leqslant R_{c} \Rightarrow j \rightarrow k \in \bigcup_{i=1}^{C} \mathcal{S}_{i} \text { and } k \rightarrow j \in \bigcup_{i=1}^{C} \mathcal{S}_{i} \\
\forall 1 \leqslant j<k \leqslant N
\end{array}
$$

A schedule $\Psi(\cdot)$ is conflict-free in terms of SINR, if the SINR at every intended receiver does not drop below the communication threshold.

$$
\operatorname{SINR}_{r_{i, j}} \geqslant \gamma_{c} \forall i=1, \ldots, C, \forall j=1, \ldots, M_{i}
$$

\section{B. Graph-Based Scheduling}

The traditional approach in designing reuse schedules is to use a graph model of the network and study the set of edges [7] [9]. The STDMA network $\Phi(\cdot)$ is modeled by a directed graph $\mathcal{G}(\mathcal{V}, \mathcal{E})$, where $\mathcal{V}$ is the set of vertices and $\mathcal{E}$ is the set of edges. Let $\mathcal{V}=\left\{v_{1}, v_{2}, \ldots, v_{N}\right\}$, where vertex $v_{j}$ represents the $j^{t h}$ node in $\Phi(\cdot)$. In general, $\mathcal{E}=\mathcal{E}_{c} \cup \mathcal{E}_{i}$, where $\mathcal{E}_{c}$ and $\mathcal{E}_{i}$ denote the set of communication and interference edges respectively. If node $k$ is within node $j$ 's communication range, then there is a communication edge from $v_{j}$ to $v_{k}$, denoted by $v_{j} \stackrel{c}{\rightarrow} v_{k}$. If node $k$ is outside node $j$ 's communication range but within its interference range, then there is an interference edge from $v_{j}$ to $v_{k}$, denoted by $v_{j} \stackrel{i}{\rightarrow} v_{k}$. Thus, the mapping from $\Phi(\cdot)$ to $\mathcal{G}(\cdot)$ can be described as follows:

$$
\begin{aligned}
D(j, k) \leqslant R_{c} & \Rightarrow \quad v_{j} \stackrel{c}{\rightarrow} v_{k} \in \mathcal{E}_{c} \text { and } v_{k} \stackrel{c}{\rightarrow} v_{j} \in \mathcal{E}_{c} \\
R_{c}<D(j, k) \leqslant R_{i} & \Rightarrow \quad v_{j} \stackrel{i}{\rightarrow} v_{k} \in \mathcal{E}_{i} \text { and } v_{k} \stackrel{i}{\rightarrow} v_{j} \in \mathcal{E}_{i}
\end{aligned}
$$

A communication or an interference edge from $v_{j}$ to $v_{k}$ will be denoted by $v_{j} \rightarrow v_{k}$. The subgraph $\mathcal{G}_{c}\left(\mathcal{V}, \mathcal{E}_{c}\right)$ consisting of communication edges only is termed as the communication graph.

The schedule $\Psi(\cdot)$ is then designed from the graph $\mathcal{G}(\cdot)$. Specifically, an STDMA link scheduling algorithm is equivalent to assigning a unique color to every communication edge in the graph, such that source-destination pairs corresponding to communication edges with the same color transmit simultaneously in a particular time slot. The traditional method 
for link assignment requires that two communication edges $v_{i} \stackrel{c}{\rightarrow} v_{j}$ and $v_{k} \stackrel{c}{\rightarrow} v_{l}$ can be colored the same iff:

i) vertices $v_{i}, v_{j}, v_{k}, v_{l}$ are all mutually distinct, i.e., there is no primary edge conflict, and

ii) $v_{i} \rightarrow v_{l} \notin \mathcal{G}(\cdot)$ and $v_{k} \rightarrow v_{j} \notin \mathcal{G}(\cdot)$, i.e, there is no secondary edge conflict.

The first criterion is based on the operational constraint. The second criterion states that a node cannot receive a packet while neighboring nodes are transmitting.

Graph-Based scheduling algorithms utilize various graph coloring methodologies to obtain a non-conflicting schedule, i.e., a schedule devoid of primary and secondary edge conflicts. To maximize the throughput of an STDMA network, graphbased scheduling algorithms seek to minimize the total number of colors used to color all the communication edges of $\mathcal{G}(\cdot)$.

\section{Limitations of Graph-Based Scheduling Algorithms}

Observe that Criteria ii) and (iii) are not sufficient to guarantee that the resulting schedule $\Psi(\cdot)$ is conflict-free. The link assignments that fulfill the above criteria do not necessarily satisfy the SINR condition (9).

Importantly, graph-based scheduling algorithms do not maximize the throughput of an STDMA network because:

1) Due to hard-thresholding based on communication and interference radii, graph-based scheduling algorithms can lead to high cumulative interference at a receiver [7] [9]. This is because the SINR at receiver $r_{i, j}$ decreases with an increase in the number of concurrent transmissions $M_{i}$, while $R_{c}$ and $R_{i}$ have been defined for a single transmission only. For example, consider

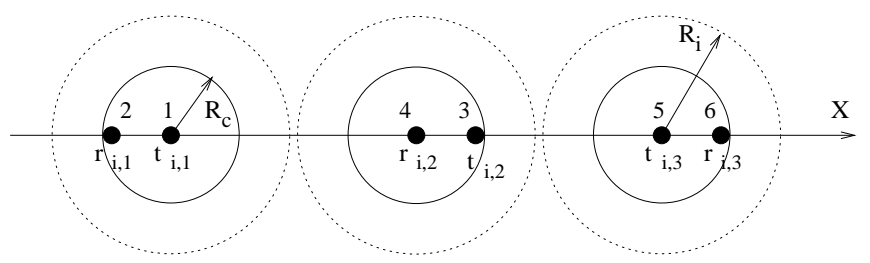

Fig. 1. Graph-Based algorithms can lead to high cumulative interference.

Figure 1 with six labeled nodes whose coordinates are $1 \equiv(-360,0), 2 \equiv(-450,0), 3 \equiv(90,0), 4 \equiv(0,0)$, $5 \equiv(360,0)$ and $6 \equiv(450,0)$. The system parameters are $P=10 \mathrm{~mW}, \alpha=4, N_{0}=-90 \mathrm{dBm}, \gamma_{c}=20$ $\mathrm{dB}$ and $\gamma_{i}=10 \mathrm{~dB}$, which yields $R_{c}=100 \mathrm{~m}$ and $R_{i}=177.8 \mathrm{~m}$. A graph-based scheduling algorithm will typically schedule the transmissions $1 \rightarrow 2,3 \rightarrow 4$ and $5 \rightarrow 6$ in the same time slot, say the $i^{t h}$ time slot, since the resulting graph coloring is devoid of primary and secondary edge conflicts. However, our computations show that the SINRs at receivers $r_{i, 1}, r_{i, 2}$ and $r_{i, 3}$ are $21.26 \mathrm{~dB}, 18.42 \mathrm{~dB}$ and $19.74 \mathrm{~dB}$ respectively. From the physical interference model, transmission $t_{i, 1} \rightarrow r_{i, 1}$ is successful, while transmissions $t_{i, 2} \rightarrow r_{i, 2}$ and $t_{i, 3} \rightarrow$ $r_{i, 3}$ are unsuccessful. This leads to low throughput.

2) On the other hand, graph-based scheduling algorithms can be extremely conservative and result in a higher

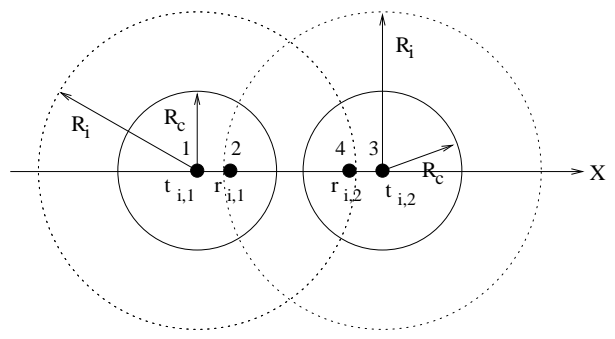

Fig. 2. Graph-Based algorithms can lead to higher number of colors.

number of colors [19]. For example, with the same system parameters as in 10, consider Figure 2] with four labeled nodes whose coordinates are $1 \equiv(0,0)$, $2 \equiv(50,0), 3 \equiv(220,0)$ and $4 \equiv(170,0)$. Assume there are two transmission requests: $1 \rightarrow 2$ and $3 \rightarrow 4$. If both the transmissions are scheduled in the same slot, say the $i^{t h}$ time slot, our computations show that the SINRs at receivers $r_{i, 1}$ and $r_{i, 2}$ are both equal to $20.91 \mathrm{~dB}$. From the physical interference model, both transmissions $t_{i, 1} \rightarrow r_{i, 1}$ and $t_{i, 2} \rightarrow r_{i, 2}$ are successful, since signals levels are so high at the receivers that strong interferences can be tolerated. However, due to secondary edge conflicts, a graph-based scheduling algorithm will schedule the above transmissions in different slots, thus decreasing the throughput.

3) Graph-based scheduling algorithms are not geographyaware, i.e., they determine a schedule without being cognizant of the exact positions of the transmitters and receivers.

\section{Problem Formulation}

In STDMA, we construct a graph model $\mathcal{G}\left(\mathcal{V}, \mathcal{E}_{c} \cup \mathcal{E}_{i}\right)$ of the physical network $\Phi(\cdot)$. The communication graph $\mathcal{G}_{c}\left(\mathcal{V}, \mathcal{E}_{c}\right)$ is an approximation of $\Phi(\cdot)$, while the two-tier graph $\mathcal{G}\left(\mathcal{V}, \mathcal{E}_{c} \cup \mathcal{E}_{i}\right)$ is a better approximation of $\Phi(\cdot)$. From $\Phi(\cdot)$ and $\mathcal{G}_{c}(\cdot)$, one can exhaustively determine the STDMA schedule which yields the highest throughput according to the physical interference model. However, this is a combinatorial optimization problem of prohibitive complexity $\left(O\left(\left|\mathcal{E}_{c}\right|^{\left|\mathcal{E}_{c}\right|}\right)\right)$ and is thus computationally infeasible.

To overcome these problems, we propose a new suboptimal algorithm for STDMA link scheduling based on a more realistic physical interference model. Our algorithm is based on the communication graph model $\mathcal{G}_{c}\left(\mathcal{V}, \mathcal{E}_{c}\right)$ as well as SINR computations.

To evaluate the performance of our algorithm and compare it with existing suboptimal STDMA link scheduling algorithms, we define the following metric: spatial reuse. Consider the STDMA link schedule $\Psi(\cdot)$ for the network $\Phi(\cdot)$. Under the physical interference model, the transmission $t_{i, j} \rightarrow r_{i, j}$ is successful iff (3) is satisfied. The spatial reuse of the schedule $\Psi(\cdot)$ is defined as the average number of successfully received packets per time slot in the STDMA schedule. Thus

$$
\text { Spatial Reuse }=\sigma=\frac{\sum_{i=1}^{C} \sum_{j=1}^{M_{i}} I\left(\operatorname{SINR}_{r_{i, j}} \geqslant \gamma_{c}\right)}{C}
$$


where $I(A)$ denote the indicator function for event $A$, i.e., $I(A)=1$ if event $A$ occurs, $I(A)=0$ if event $A$ does not occur.

The essence of STDMA is to have a reasonably large number of concurrent and successful transmissions. For an STDMA network which is operational for a long period of time, say $L$ slots, the total number of successfully received packets is $L \sigma$. Thus, a high value of spatial reuse 3 directly translates to higher long-term total network throughput and the number of colors $C$ is relatively unimportant. Hence, spatial reuse turns out to be a crucial metric for the comparison of different STDMA algorithms.

We seek low complexity conflict-free STDMA link scheduling algorithms with high spatial reuse. We only consider STDMA schedules which are feasible and exhaustive. Thus, our schedules satisfy (6), (7), (8) and (9).

\section{E. Comparison with SINR-Based Scheduling Algorithms}

In cognizance of our system model and performance metric, our work is reasonably different from existing SINR-based STDMA link scheduling algorithms.

The works in [11] [12] focus on minimizing the schedule length, which does not necessarily translate to high network throughput. On the other hand, spatial reuse directly corresponds to network throughput capacity [18]. Power-controlled algorithms can lead to excessively high transmit power (for example, Line 16 in Algorithm 1 [12]), which is impractical since all wireless routers have constraints on maximum transmit power. So, similar to [17], we consider uniform transmit power at all wireless routers. Existing works on SINR-based link scheduling, which are usually in the context of 802.11 wireless networks [11] [15] and wireless mesh networks [16] [17], consider many practical aspects of the underlying communication protocol and network architecture. Consequently, their system models are quite different from our pure STDMA network model.

To the best of our knowledge, this is the first attempt to develop a centralized algorithm for SINR-based link scheduling in a pure STDMA wireless ad hoc network with uniform power assignment. Also, this work is different from previous works due to the focus on spatial reuse.

Hence, we compare the performance of our algorithm with existing graph-based algorithms only.

\section{SINR-BASEd Link SchedUling Algorithm}

\section{A. Structure}

We first describe the essential features of STDMA link scheduling algorithms. The core of every link scheduling algorithm consists of the following modules:

1) An order in which communication edges are considered for coloring.

2) A function which determines the set of all existing colors which can be assigned to the edge under consideration without violating the problem constraints.

\footnotetext{
${ }^{3}$ Note that spatial reuse in our system model is analogous to spectral efficiency in digital communication systems.
}

3) A BestColor rule to determine which conflict-free color to assign to the edge under consideration.

The second module considers only operational and communication range constraints in graph-based scheduling algorithms. However, in the SINR-based link scheduling algorithm that we propose, SINR constraints are also taken into account. Note that this function is completely described by the problem definition and does not vary from algorithm to algorithm. The ordering of edges for coloring and the BestColor rule play a significant role in determining the performance and computational complexity of an STDMA scheduling algorithm.

\section{B. Motivation}

Recall that graph-based models are inadequate to design efficient link schedules under the physical interference model and brute-force computation of an optimal link schedule that maximizes spatial reuse is prohibitively complex (see Sections II-C and [I-D). Motivated by techniques from matroid theory [20], we develop a computationally feasible algorithm with demonstrably high spatial reuse. The essence of our algorithm is to partition the set of communication edges into subsets (forests) and color the edges in each subset sequentially. The edges in each forest are considered in a random order for coloring, since randomized algorithms are known to outperform deterministic algorithms, esp. when the characteristics of the input are not known apriori [21].

A similar matroid-based network partitioning technique is used in [22] to generate high capacity subnetworks for a distributed throughput maximization problem in wireless mesh networks. Techniques from matroid theory have also been employed to develop efficient heuristics for NP-hard combinatorial optimization problems in fields such as distributed computer systems [23] and linear network theory [24].

\section{ConflictFreeLinkSchedule Algorithm}

Our proposed SINR-based link scheduling algorithm is ConflictFreeLinkSchedule, which considers the communication graph $\mathcal{G}_{c}\left(\mathcal{V}, \mathcal{E}_{c}\right)$ and is described in Algorithm 1

In Phase 1 (Line 3), we label all the vertices randomly. Specifically, if $\mathcal{G}_{c}(\cdot)$ has $v$ vertices, we perform a random permutation of the sequence $(1,2, \ldots, v)$ and assign these labels to vertices with indices $1,2, \ldots, v$ respectively.

In Phase 2 (Line 4$)$, the communication graph $\mathcal{G}_{c}(\cdot)$ is decomposed into what are called as out-oriented and in-oriented graphs $T_{1}, T_{2}, \ldots, T_{k}$ [1]. Each $T_{i}$ is a forest and every edge of $\mathcal{G}_{c}(\cdot)$ is in exactly one of the $T_{i}$ 's. This decomposition is achieved by partitioning graph $G_{c}(\cdot)$, the undirected equivalent of $\mathcal{G}_{c}(\cdot)$, into undirected forests. The number of forests can be minimized by using techniques from Matroid theory ( $k$ forest problem, [25]). However, this optimal decomposition requires extensive computation. Hence, we adopt the speedier albeit non-optimal approach of using successive breadth first searches to decompose $G_{c}(\cdot)$ into undirected forests. Each undirected forest is further mapped to two directed forests. In one forest, the edges in every connected component point away from the root and every vertex has at most one incoming edge, thus producing an out-oriented graph. In the other forest, the 
edges in every connected component point toward the root and every vertex has at most one outgoing edge, thus producing an in-oriented graph 4 .

In Phase 3 (Lines 5-14), the oriented graphs are considered sequentially. For each oriented graph, vertices are considered in increasing order by labe 5 and the unique edge associated with each vertex is colored using the FirstConflictFreeColor function.

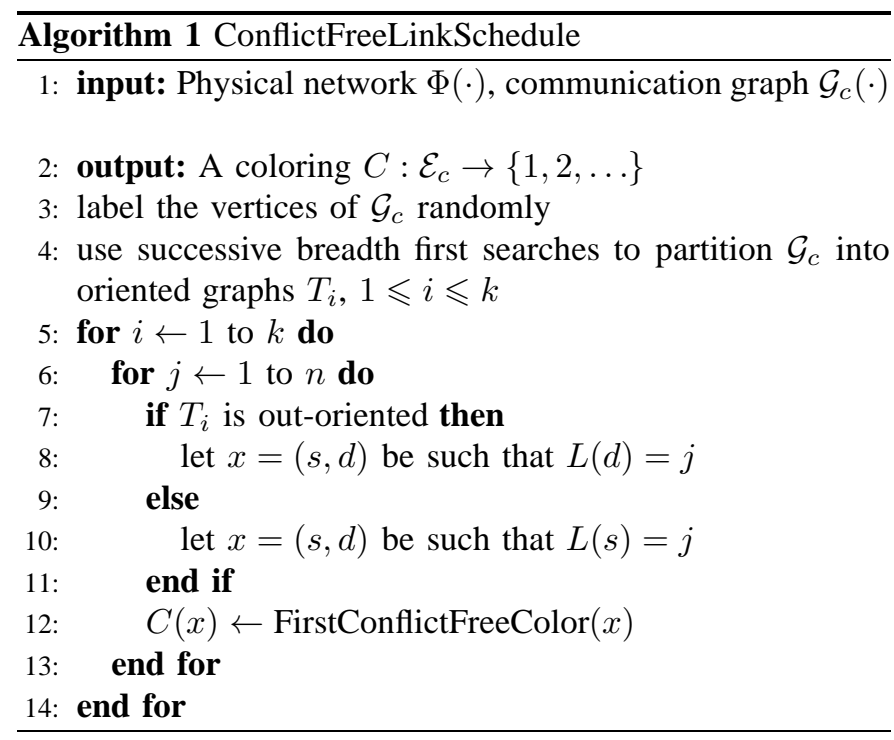

The FirstConflictFreeColor function is explained in Algorithm 2, For the edge under consideration $x$, it discards any color that has an edge with a primary conflict with $x$. Now, we consider the residual set of conflict-free colors sequentially. We choose the first conflict-free color such that the resulting SINRs at the receiver of $x$ and the receivers of all co-colored edges exceed the communication threshold $\gamma_{c}$. If no such color is found, we assign a new color to $x$. Thus, this function guarantees that the ensuing schedule is conflict-free in terms of SINR. Since we choose the first SINR-compliant color and not the 'best' SINR-compliant color according to some BestColor rule, the computational complexity of this function is lower than that of any other function which checks the SINRs at the receivers of all co-colored edges.

\section{Performance Results}

\section{A. Simulation Model}

In our simulation experiments, the location of every node is generated randomly, using a uniform distribution for its $X$ and $Y$ coordinates, in the deployment area. For a fair comparison of our algorithm with the Truncated Graph-Based Scheduling Algorithm [7], we assume that the deployment region is a circular region of radius $R$. Thus, if $\left(X_{j}, Y_{j}\right)$ are the Cartesian coordinates of the $j^{\text {th }}$ node, $j=1, \ldots, N$, then $X_{j} \sim U[-R, R]$ and $Y_{j} \sim U[-R, R]$ subject to $X_{j}^{2}+Y_{j}^{2} \leqslant$ $R^{2}$. Equivalently, if $\left(R_{j}, \Theta_{j}\right)$ are the polar coordinates of

\footnotetext{
${ }^{4} \mathrm{An}$ in-oriented graph is also constructed by Algorithm 1 in [12] to determine a link schedule in a power-controlled STDMA network.

${ }^{5}$ In essence, the edges are scanned in a random order, since labeling is random.
}

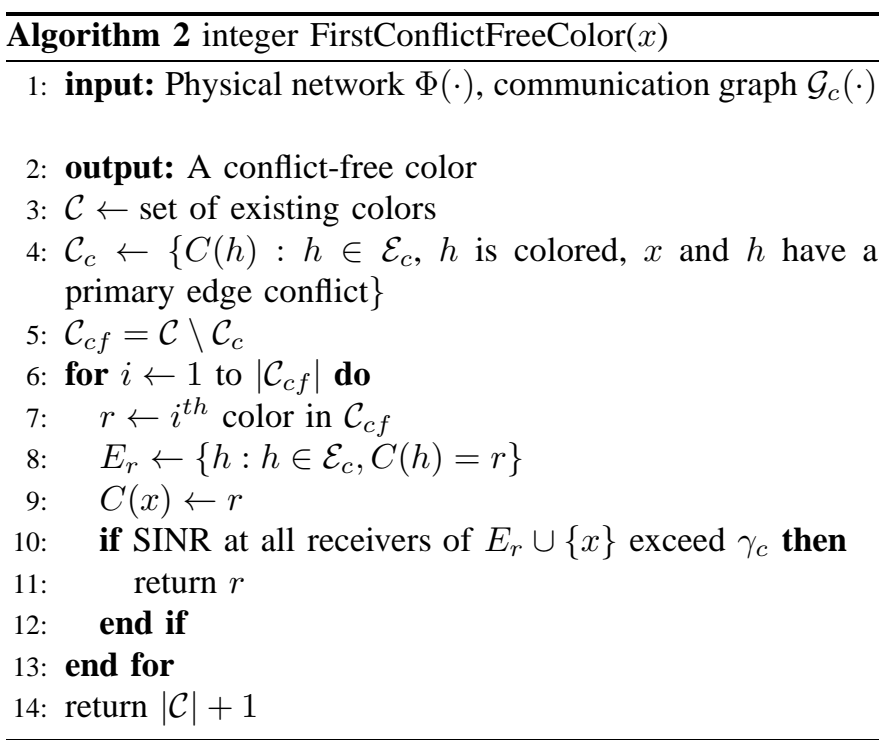

the $j^{\text {th }}$ node, then $R_{j}^{2} \sim U\left[0, R^{2}\right]$ and $\Theta_{j} \sim U[0,2 \pi]$. After generating random positions for $N$ nodes, we have complete information of $\Phi(\cdot)$. Using (4) and (5), we compute the communication and interference radii, and then map the network $\Phi(\cdot)$ to the two-tier graph $\mathcal{G}\left(\mathcal{V}, \mathcal{E}_{c} \cup \mathcal{E}_{i}\right)$. Once the link schedule is computed by every algorithm, the spatial reuse is computed using (10). We use two sets of values for system parameters $P, \gamma_{c}, \gamma_{i}, \alpha, N_{0}, N$ and $R$, which are prototypical values of system parameters in wireless networks [15] and describe them in Section IV-B For a given set of system parameters, we calculate the spatial reuse by averaging this quantity over one thousand randomly generated networks. Keeping all other parameters fixed, we observe the effect of increasing the number of nodes $N$ on the spatial reuse $\sigma$.

In our experiments, we compare the performance of the following algorithms:

1) ArboricalLinkSchedule [1] (ALS)

2) Truncated Graph-Based Scheduling Algorithm6 [7] (TGSA)

3) ConflictFreeLinkSchedule (CFLS)

\section{B. Performance Comparison}

In our first set of experiments (Experiment 1), we assume that $R=500 \mathrm{~m}, P=10 \mathrm{~mW}, \alpha=4, N_{0}=-90 \mathrm{dBm}, \gamma_{c}=$ $20 \mathrm{~dB}$ and $\gamma_{i}=10 \mathrm{~dB}$. Thus, $R_{c}=100 \mathrm{~m}$ and $R_{i}=177.8$ $\mathrm{m}$. We vary the number of nodes from 30 to 110 in steps of 5 . Figure 3 plots the spatial reuse vs. number of nodes for all the algorithms.

In our second set of experiments (Experiment 2), we assume that $R=700 \mathrm{~m}, P=15 \mathrm{~mW}, \alpha=4, N_{0}=-85 \mathrm{dBm}, \gamma_{c}=$ $15 \mathrm{~dB}$ and $\gamma_{i}=7 \mathrm{~dB}$. Thus, $R_{c}=110.7 \mathrm{~m}$ and $R_{i}=175.4$

\footnotetext{
${ }^{6}$ In Truncated Graph-Based Scheduling Algorithm, for the computation of optimal number of transmissions $M^{*}$, we follow the exact method described in [7]. Since $0<\xi<\frac{N_{0}}{P}$, we assume that $\xi=0.9999 \frac{N_{0}}{P}$ and compute successive Edmundson-Madansky (EM) upper bounds [26] [27] till the difference between successive EM bounds is less than $0.3 \%$. We have experimentally verified that only high values of $\xi$ lead to reasonable values for $M^{*}$, whereas low values of $\xi$, say $\xi=0.1 \frac{N_{0}}{P}$, lead to the extremely conservative value of $M^{*}=1$ in most cases.
} 


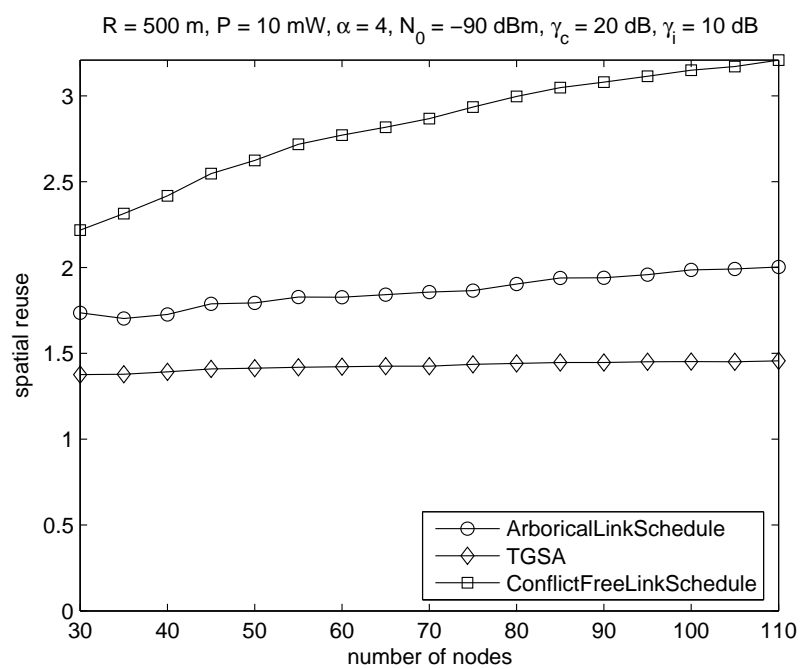

Fig. 3. Spatial reuse vs. number of nodes for Experiment 1 .

$\mathrm{m}$. We vary the number of nodes from 70 to 150 in steps of 5 . Figure 4 plots the spatial reuse vs. number of nodes for all the algorithms.

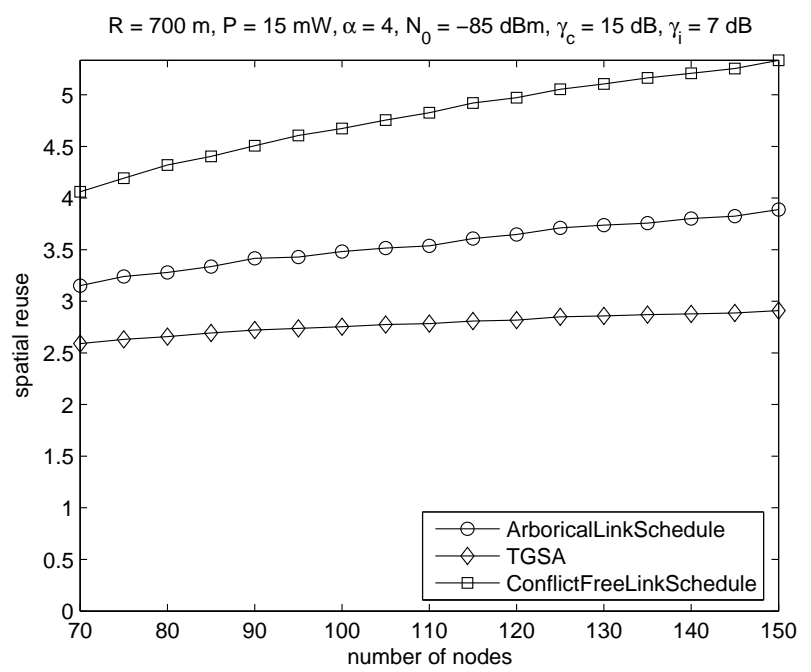

Fig. 4. Spatial reuse vs. number of nodes for Experiment 2.

For the ALS algorithm, we observe that spatial reuse increases very slowly with increasing number of nodes.

For the TGSA algorithm, we observe that spatial reuse is 18$27 \%$ lower than that of ALS. A plausible explanation for this behavior is as follows. The basis for TGSA is the computation of $M^{*}$, the optimal number of transmissions in every slot. $M^{*}$ is determined by maximizing a lower bound on the expected number of successful transmissions in a time slot. Since the partitioning of a maximal independent set of communication arcs into subsets of cardinality at most $M^{*}$ is arbitrary and not geography-based, there could be scenarios where the transmissions scheduled in a subset are in the vicinity of each other, resulting in moderate to high interference. In essence, maximizing this lower bound does not necessarily translate to maximizing the number of successful transmissions in a time slot. Also, due to its design, the TGSA algorithm yields higher number of colors compared to ALS.

For our CFLS algorithm, we observe that spatial reuse increases steadily with increasing number of nodes and is consistently $25-50 \%$ higher than the spatial reuse of ALS and TGSA.

\section{Performance Comparison under Realistic Channel Condi- tions}

In a realistic wireless environment, channel impairments like multipath fading and shadowing affect the received SINR at a receiver [28]. In this section, we compare the performance of the ALS, TGSA and CFLS algorithms in a wireless channel which experiences Rayleigh fading and lognormal shadowing.

In the absence of fading and shadowing, the SINR at receiver $r_{i, j}$ is given by (1). We assume that every algorithm (ALS, TGSA and CFLS) considers only path loss in the channel prior to constructing the two-tier graph $\mathcal{G}\left(\mathcal{V}, \mathcal{E}_{c} \cup \mathcal{E}_{i}\right)$ and computing the link schedule. However, when evaluating the performance of each algorithm, we take into account the fading and shadowing channel gains between every pair of nodes. Specifically, for computing the spatial reuse using (10), we assume that the (actual) SINR at receiver $r_{i, j}$ is given by

$$
\operatorname{SINR}_{r_{i, j}}=\frac{\frac{P}{D^{\alpha}\left(t_{i, j}, r_{i, j}\right)} V\left(t_{i, j}, r_{i, j}\right) 10^{W\left(t_{i, j}, r_{i, j}\right)}}{N_{0}+\sum_{\substack{k=1 \\ k \neq j}}^{M_{i}} \frac{P}{D^{\alpha}\left(t_{i, k}, r_{i, j}\right)} V\left(t_{i, k}, r_{i, j}\right) 10^{W\left(t_{i, k}, r_{i, j}\right)}}
$$

where random variables $V(\cdot)$ and $W(\cdot)$ correspond to channel gains due to Rayleigh fading and lognormal shadowing respectively. We assume that $\{V(k, l) \mid 1 \leqslant k, l \leqslant N, k \neq$ $l\}$ are independent and identically distributed (i.i.d.) random variables with probability density function 7 (pdf) [29]

$$
f_{V}(v)=\frac{1}{\sigma_{V}^{2}} e^{\frac{-v}{\sigma_{V}^{2}}} u(v)
$$

and $\{W(k, l) \mid 1 \leqslant k, l \leqslant N, k \neq l\}$ are i.i.d. zero mean Gaussian random variables with pdf [30]

$$
f_{W}(w)=\frac{1}{\sqrt{2 \pi} \sigma_{W}} e^{\frac{-w^{2}}{2 \sigma_{W}^{2}}}
$$

Random variables $V(\cdot)$ and $W(\cdot)$ are independent of each other and also independent of the node locations.

Our simulation model and experiments are exactly as described in Sections IV-A and IV-B. In our simulations, we assume $\sigma_{V}^{2}=\sigma_{W}^{2}=1$. For Experiment 1, Figure 5 plots the spatial reuse vs. number of nodes for all the algorithms. For Experiment 2, Figure 6plots spatial reuse vs. number of nodes for all the algorithms.

From Figures 3, 4, 5, and 6, we observe that spatial reuse decreases by $20-40 \%$ in a channel experiencing multipath fading and shadowing effects. A plausible explanation for this observation is as follows. Since the channel gains between every pair of nodes are independent of each other, it is reasonable to assume that the interference power at a typical receiver remains almost the same as in the non-fading case. This is

\footnotetext{
${ }^{7} u(\cdot)$ is the unit step function.
} 


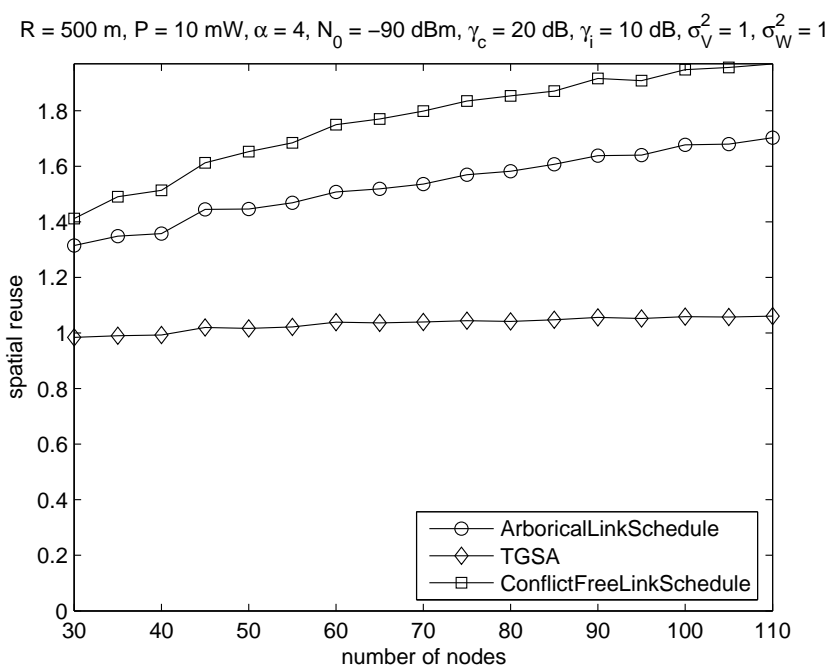

Fig. 5. Spatial reuse vs. number of nodes for Experiment 1 under multipath fading and shadowing channel conditions.

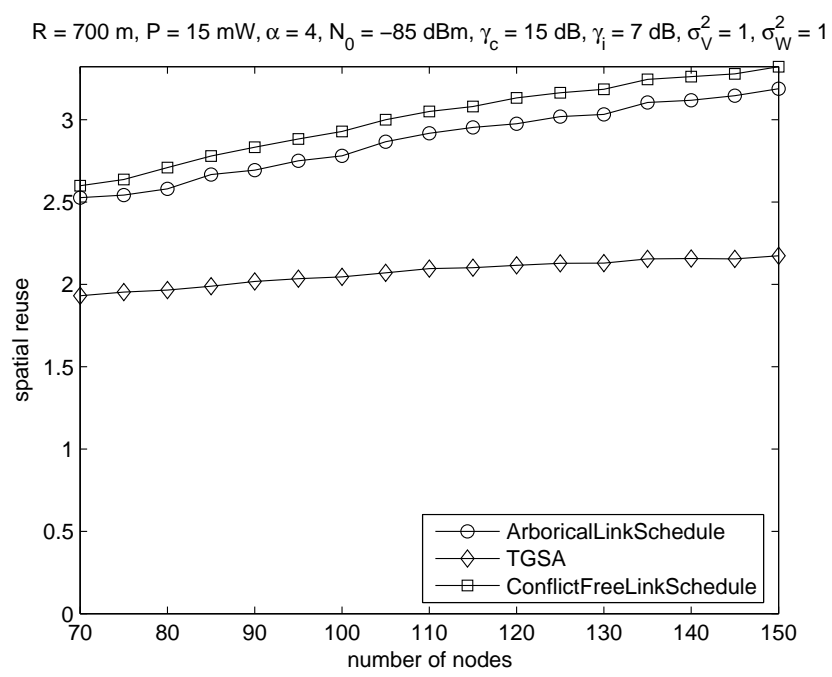

Fig. 6. Spatial reuse vs. number of nodes for Experiment 2 under multipath fading and shadowing channel conditions.

because, even if the power received from few unintended transmitters is low, the power received from other unintended transmitters will be high (on an average); thus the interference power remains constant. Consequently, the change in SINR is determined by the change in received signal power only. If the received signal power is higher compared to the nonfading case, the transmission is anyway successful and spatial reuse remains unchanged (see (10)). However, if the received signal power is lower, the transmission is now unsuccessful and spatial reuse decreases. Hence, on an average, the spatial reuse decreases.

Finally, from Figures 5 and 6, we observe that our CFLS algorithm achieves $5-17 \%$ higher spatial reuse than the ALS algorithm and $40-80 \%$ higher spatial reuse than the TGSA algorithm, under realistic channel conditions.

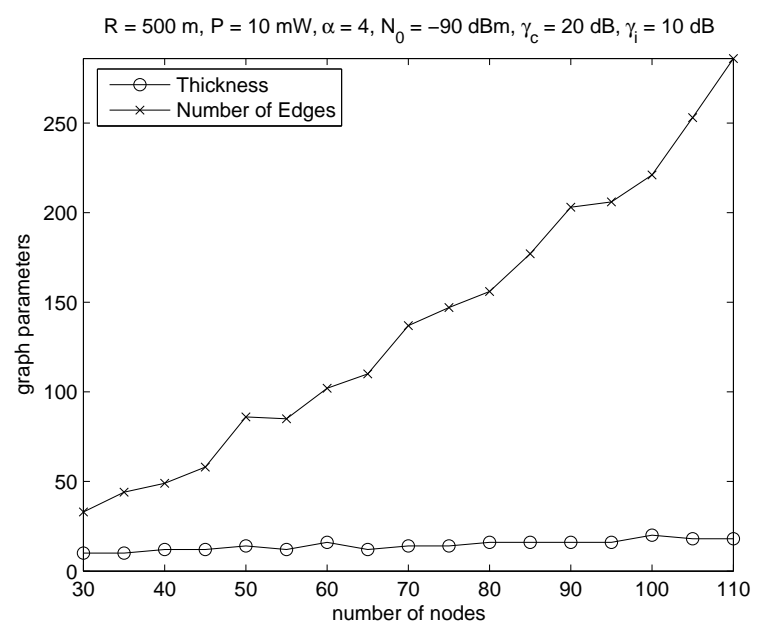

(a) Experiment 1

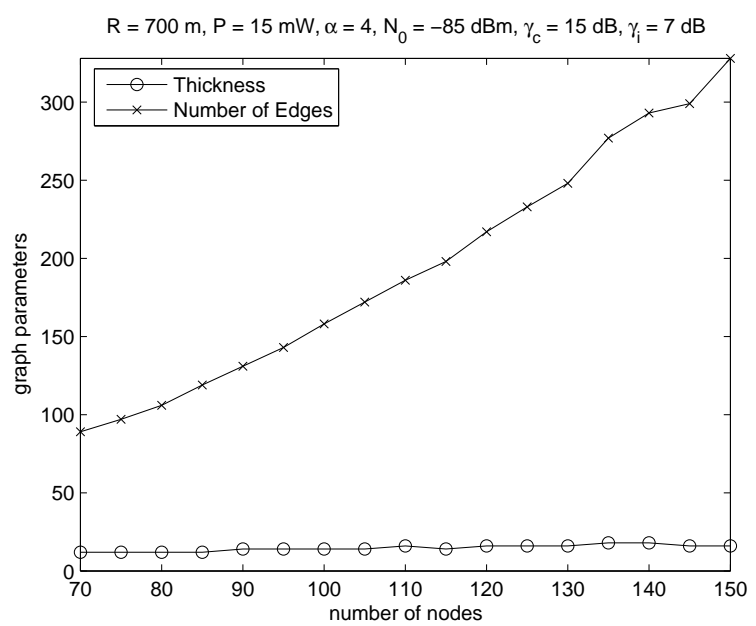

(b) Experiment 2

Fig. 7. Comparison of thickness and number of edges with number of vertices.

\section{Analytical Results}

In this section, we derive upper bounds on the running time complexity (computational complexity) of the ConflictFreeLinkSchedule algorithm. We will use the following notation with respect to the communication graph $\mathcal{G}_{c}\left(\mathcal{V}, \mathcal{E}_{c}\right)$ :

$$
\begin{aligned}
e= & \text { number of communication edges } \\
v= & \text { number of vertices } \\
\theta= & \text { thickness of the graph } \\
:= & \text { minimum number of graphs into which the } \\
& \text { undirected equivalent of } \mathcal{G}_{c}(\cdot) \text { can be partitioned }
\end{aligned}
$$

Before we prove our results, it is instructive to observe Figure 7, which shows the variation of $\theta$ and $e$ with $v$ for the two experiments described in Section IV-B. Since determining the thickness of a graph is NP-hard [31], each value of $\theta$ in Figure 7 is an upper bound on the actual thickness based on the number of forests into which the undirected equivalent of the communication graph has been decomposed using successive breadth first searches. We observe that the graph thickness 
increases very slowly with the number of vertices, while the number of edges increases super-linearly with the number of vertices.

Lemma 1: An oriented graph $T$ can be colored using no more than $O(v)$ colors using ConflictFreeLinkSchedule.

Proof: Since an oriented graph with $v$ vertices has at most $v$ edges, the edges of $T$ can be colored with at most $v$ colors.

Remark 1: The number of colors obtained by our algorithm depends not only on the graph representation of the STDMA network, but also on the positions of the nodes and the values of $P, \gamma_{c}, \gamma_{i}, \alpha$ and $N_{0}$. Since our algorithm is both graphbased and SINR-based (hybrid), it is not possible to obtain a tighter upper bound in Lemma 1.

Lemma 2: For an oriented graph $T$, the running time of ConflictFreeLinkSchedule is $O\left(v^{2}\right)$.

Proof: Assuming that an element can be chosen randomly and uniformly from a finite set in unit time (Chapter 1, [21]), the running time of Phase 1 can be shown to be $O(v)$. Since there is only one oriented graph, Phase 2 runs in time $O(1)$. In Phase 3, the unique edge associated with the vertex under consideration is assigned a color using FirstConflictFreeColor. From Lemma 1 , the size of the set of colors to be examined $\left|\mathcal{C}_{c} \cup \mathcal{C}_{c f}\right|$ is $O(v)$. In FirstConflictFreeColor, the SINR is checked only once for every colored edge in the set $\bigcup_{i=1}^{\left|\mathcal{C}_{c f}\right|} E_{i}$ and at most $v$ times for the edge under consideration $x$. With a careful implementation, FirstConflictFreeColor runs in time $O(v)$. So, the running time of Phase 3 is $O\left(v^{2}\right)$. Thus, the total running time is $O\left(v^{2}\right)$.

Theorem 1: For an arbitrary graph $\mathcal{G}$, the running time of ConflictFreeLinkSchedule is $O(e v \log v+e v \theta)$.

Proof: Assuming that an element can be chosen randomly and uniformly from a finite set in unit time [21], the running time of Phase 1 can be shown to be $O(v)$. For Phase 2, the optimal partitioning technique of [25] based on Matroids can be used to partition the communication graph $\mathcal{G}_{c}$ into at most $6 \theta$ oriented graphs in time $O(e v \log v)$. Thus, $k \leqslant 6 \theta$ holds for Phase 3. From Lemma 2, it follows that the first oriented graph $T_{1}$ can be colored in time $O\left(v^{2}\right)$. However, consider the coloring of the $j^{\text {th }}$ oriented graph $T_{j}$, where $2 \leqslant j \leqslant k$. When coloring edge $x$ from $T_{j}$ using FirstConflictFreeColor, conflicts can occur not only with the colored edges of $T_{j}$, but also with the edges of the previously colored oriented graphs $T_{1}, T_{2}, \ldots, T_{j-1}$. This fact is exemplified in Appendix [ Hence, the worst-case size of the set of colors to be examined $\left|\mathcal{C}_{c} \cup \mathcal{C}_{c f}\right|$ is $O(e)$. Note that in FirstConflictFreeColor, the SINR is checked only once for every colored edge in the set $\bigcup_{i=1}^{\left|\mathcal{C}_{c f}\right|} E_{i}$ and at most $e$ times for the edge under consideration $x$. With a careful implementation, FirstConflictFreeColor runs in time $O(e)$. Hence, any subsequent oriented graph $T_{j}$ can be colored in time $O(e v)$. Thus, the running time of Phase 3 is $O(e v \theta)$. Therefore, the overall running time of ConflictFreeLinkSchedule is $O(e v \log v+e v \theta)$.

\section{DISCUSSION}

In this paper, we have developed ConflictFreeLinkSchedule, an SINR-based link scheduling algorithm for STDMA multihop wireless ad hoc networks under the physical interference model. The performance of our algorithm is superior to existing link scheduling algorithms for STDMA networks with uniform power assignment. A practical experimental modeling shows that, on an average, our algorithm achieves $40 \%$ higher spatial reuse than the ArboricalLinkSchedule [1] and Truncated Graph-Based Scheduling [7] algorithms. Since schedules are constructed offline only once and then used by the network for a long period of time, these improvements in performance directly translate to higher long-term network throughput.

The computational complexity of ConflictFreeLinkSchedule is comparable to the computational complexity of ArboricalLinkSchedule and is much lower than the computational complexity of Truncated Graph-Based Scheduling Algorithm. Thus, in cognizance of spatial reuse as well as computational complexity, ConflictFreeLinkSchedule is a good candidate for efficient SINR-based STDMA link scheduling algorithms.

We have recently developed computationally efficient algorithms for STDMA broadcast scheduling under the physical interference model. It would be interesting to apply techniques like simulated annealing, genetic algorithms and neural networks to compute high spatial reuse conflict-free STDMA link schedules.

\section{APPENDIX I \\ EXAMPLE OF PRIMARY EDGE CONFLICTS WITH PREVIOUSLY COLORED ORIENTED GRAPHS}

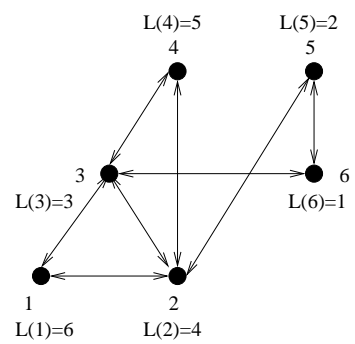

Fig. 8. A six-node STDMA wireless ad hoc network, its communication graph and node labels.

Consider the six-node STDMA wireless ad hoc network shown in Figure 8, along with its associated communication graph $\mathcal{G}_{c}(\cdot)$ and node labels. Using successive breadth first searches, $\mathcal{G}_{c}(\cdot)$ is partitioned into four oriented graphs $T_{1}, T_{2}$, $T_{3}$ and $T_{4}$, as shown in Figure 9 A conflict-free coloring of the first oriented graph $T_{1}$ is shown in Table [. Now, when we color an edge from any other oriented graph, we must take into account the colors of the edges in $T_{1}$. For example:

1) In $T_{2}$, Edge $6 \rightarrow 3$ cannot be assigned Color 2 due to a primary edge conflict with Edge $1 \rightarrow 3$ of $T_{1}$.

2) In $T_{3}$, Edge $5 \rightarrow 6$ cannot be assigned Color 1 due to a primary edge conflict with Edge $2 \rightarrow 5$ of $T_{1}$.

3) In $T_{4}$, Edge $3 \rightarrow 2$ cannot be assigned Color 3 due to a primary edge conflict with Edge $1 \rightarrow 2$ of $T_{1}$. 

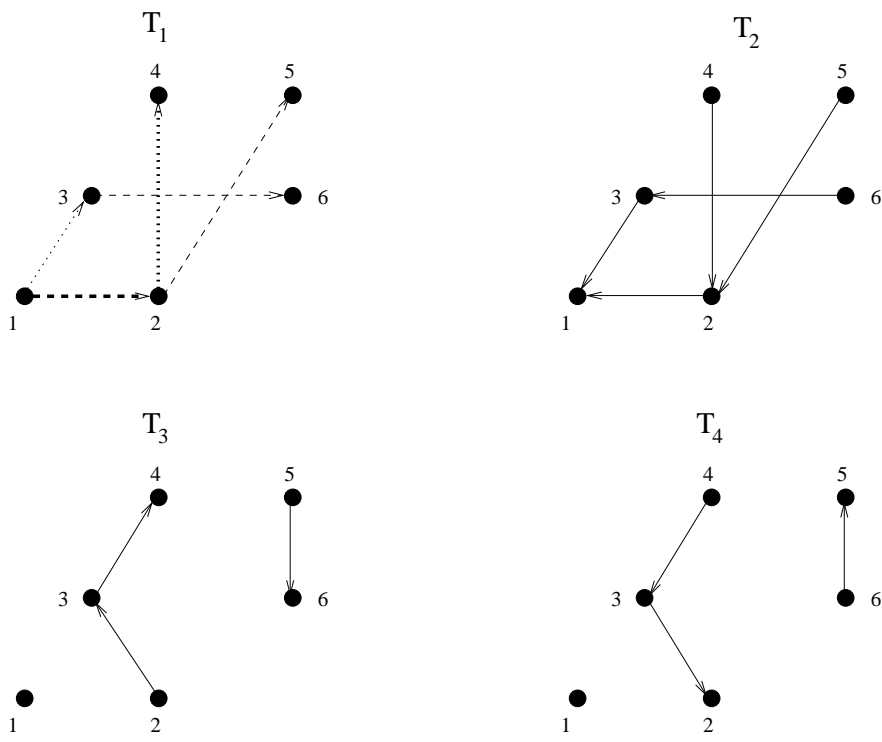

Fig. 9. Decomposition of Fig. 8 into two out-oriented graphs $\left(T_{1}, T_{3}\right)$ and two in-oriented graphs $\left(T_{2}, T_{4}\right)$.

\begin{tabular}{|c|c|}
\hline Edge of $T_{1}$ & Color \\
\hline $3 \rightarrow 6$ & 1 \\
\hline $2 \rightarrow 5$ & 1 \\
\hline $1 \rightarrow 3$ & 2 \\
\hline $1 \rightarrow 2$ & 3 \\
\hline $2 \rightarrow 4$ & 4 \\
\hline
\end{tabular}

TABLE I

CONFLICT-FREE COLORING OF FIRST ORIENTED GRAPH $T_{1}$.

\section{REFERENCES}

[1] S. Ramanathan and E. L. Lloyd, "Scheduling Algorithms for Multihop Radio Networks," IEEE/ACM Trans. Networking, vol. 1, no. 2, pp. 166177, April 1993.

[2] R. Nelson and L. Kleinrock, "Spatial TDMA: A Collision-Free Multihop Channel Access Protocol," IEEE Trans. Commun., vol. 33, no. 9, pp. 934-944, September 1985.

[3] B. Hajek and G. Sasaki, "Link Scheduling in Polynomial Time," IEEE Trans. Inform. Theory, vol. 34, no. 5, pp. 910-917, September 1988.

[4] N. Funabiki and Y. Takefuji, "A Parallel Algorithm for Broadcast Scheduling Problems in Packet Radio Networks," IEEE Trans. Commun., vol. 41, no. 6, pp. 828-831, June 1993.

[5] I. Chlamtac and A. Lerner, "Fair Algorithms for Maximal Link Activation in Multihop Radio Networks," IEEE Trans. Commun., vol. 35, no. 7, pp. 739-746, July 1987.

[6] I. Chlamtac and S. Pinter, "Distributed Nodes Organization Algorithm for Channel Access in a Multihop Dynamic Radio Network," IEEE Trans. Comput., vol. 36, no. 6, pp. 728-737, June 1987.

[7] A. Behzad and I. Rubin, "On the Performance of Graph-based Scheduling Algorithms for Packet Radio Networks," in IEEE GLOBECOM 2003, vol. 6, December 2003, pp. $3432-3436$.

[8] X. Guo, S. Roy, and W. S. Conner, "Spatial Reuse in Wireless Ad-hoc Networks," in IEEE VTC 2003, vol. 3, October 2003, pp. 1437-1442.

[9] J. Grönkvist and A. Hansson, "Comparison Between Graph-Based and Interference-Based STDMA Scheduling," in ACM MOBIHOC, October 2001.

[10] O. Somarriba, "Multihop Packet Radio Systems in Rough Terrain," Ph.D. dissertation, Royal Institute of Technology, Stockholm, Sweden, October 1995.

[11] G. Brar, D. M. Blough, and P. Santi, "Computationally Efficient Scheduling with the Physical Interference Model for Throughput Improvement in Wireless Mesh Networks," in ACM MobiCom 2006, Los Angeles, CA, September 2006, pp. 2-13.

[12] T. Moscibroda and R. Wattenhofer, "The Complexity of Connectivity in Wireless Networks," in IEEE INFOCOM 2006, Barcelona, Spain, April 2006.
[13] T. Moscibroda, R. Wattenhofer, and A. Zollinger, "Topology Control Meets SINR: The Scheduling Complexity of Arbitrary Topologies," in ACM MobiHoc 2006, Florence, Italy, May 2006, pp. 310-321.

[14] P. von Rickenbach, S. Schmid, R. Wattenhofer, and A. Zollinger, "The Complexity of Connectivity in Wireless Networks," in 5th Int. Workshop on Algorithms for Wireless, Mobile, Ad Hoc and Sensor Networks (WMAN), Denver, Colorado, USA, May 2005.

[15] T.-S. Kim, H. Lim, and J. C. Hou, "Improving Spatial Reuse through Tuning Transmit Power, Carrier Sense Threshold, and Data Rate in Multihop Wireless Networks," in ACM MobiCom 2006, Los Angeles, CA, September 2006, pp. 366-377.

[16] H. Lim, C. Lim, and J. C. Hou, "A Coordinate-Based Approach for Exploiting Temporal-Spatial Diversity in Wireless Mesh Networks," in ACM MobiCom 2006, Los Angeles, CA, September 2006, pp. 14-25.

[17] M. Kodialam and T. Nandagopal, "Characterizing Achievable Rates in Multi-Hop Wireless Mesh Networks With Orthogonal Channels," IEEE/ACM Trans. Networking, vol. 13, no. 4, pp. 868-880, August 2005.

[18] P. Gupta and P. R. Kumar, "The Capacity of Wireless Networks," IEEE Trans. Inform. Theory, vol. 46, pp. 388-404, March 2000.

[19] T. Moscibroda, R. Wattenhofer, and Y. Weber, "Protocol Design Beyond Graph-Based Models," in ACM HotNets-V, Irvine, CA, November 2006.

[20] E. Lawler, Combinatorial Optimization: Networks and Matroids. New York: Holt, Rinehart and Winston, 1976.

[21] R. Motwani and P. Raghavan, Randomized Algorithms. Cambridge University Press, 1995.

[22] A. Brzezinski, G. Zussman, and E. Modiano, "Enabling Distributed Throughput Maximization in Wireless Mesh Networks - A Partitioning Approach," in ACM MobiCom 2006, Los Angeles, CA, September 2006, pp. 26-37.

[23] T. Ramalingom, K. Thulasiraman, and A. Das, "A Matroid-Theoretic Solution to an Assignment Problem in the Conformance Testing of Communication Protocols," IEEE Trans. Comput., vol. 49, no. 4, pp. 317-330, April 2000.

[24] B. Petersen, "Investigating Solvability and Complexity of Linear Active Networks by Means of Matroids," IEEE Trans. Circuits Syst., vol. 26, no. 5, pp. 330-342, May 1979.

[25] H. N. Gabow and H. H. Westermann, "Forests, Frames, and Games: Algorithms for Matroid Sums and Applications," Algorithmica, vol. 7, no. 5\&6, pp. 465-497, 1992.

[26] A. Madansky, "Inequalities for Stochastic Linear Programming Problems," Management Science, vol. 6, pp. 197-204, 1960

[27] S. P. Dokov and D. P. Morton, "Higher-Order Upper Bounds on the Expectation of a Convex Function," Stochastic Programming EPrint Series, 2002, [Online] http://edoc.hu-berlin.de/series/speps/20028/PDF/8.pdf.

[28] B. Sklar, "Rayleigh Fading Channels in Mobile Digital Communication Systems Part 1: Characterization," IEEE Commun. Mag., vol. 35, no. 7, pp. 90-100, July 1997.

[29] D. Tse and P. Viswanath, Fundamentals of Wireless Communication. Cambridge University Press, 2005.

[30] A. Goldsmith, Wireless Communications. Cambridge University Press, 2005.

[31] P. Mutzel, T. Odenthal, and M. Scharbrodt, "The Thickness of Graphs: A Survey," Graphs and Combinatorics, vol. 14, pp. 59-73, 1998. 